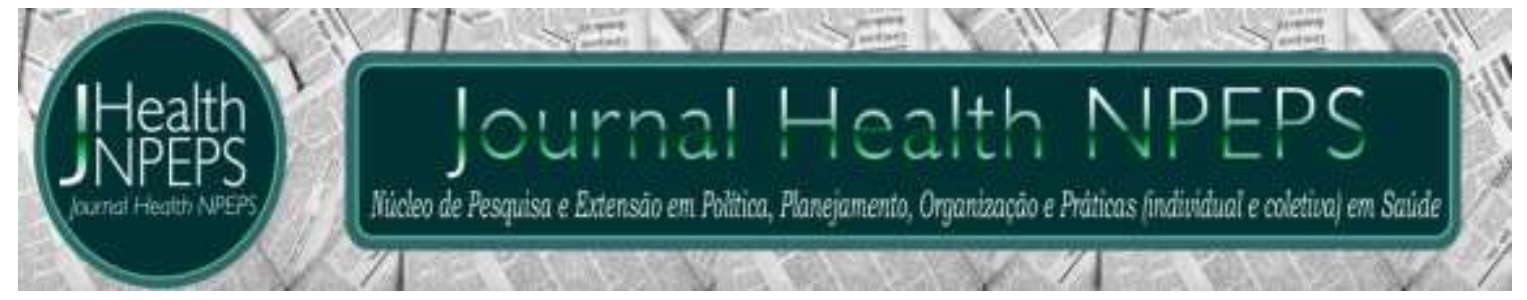

http://dx.doi.org/10.30681/252610103821

ARTIGO ORIGINAL

\title{
Perfil de comportamento e características sexuais de caminhoneiros
}

\author{
Profile of behavior and sexual characteristics of truck drivers
}

\section{Perfil de comportamiento y características sexuales de conductores de camiones}

\section{Rodrigo da Silva Busanello', Edson Antônio Silva Lima², Andre Cantarelli Vilela $^{3}$, Mariana Rodrigues Santana ${ }^{4}$, Fernando Riegel ${ }^{5}$, Alisséia Guimarães Lemes ${ }^{6}$, Elias Marcelino da Rocha ${ }^{7}$}

\section{RESUMO}

Objetivo: descrever o comportamento e os aspectos da sexualidade de caminhoneiros. Método: estudo transversal e com abordagem quantitativa junto a 177 caminhoneiros em 2018, em um município do leste de Mato Grosso. A coleta de dados foi realizada por meio de entrevista semiestruturada. Para a análise e tabulação dos dados, utilizou-se a estatística descritiva e o software Epi Info. Resultados: verificou-se que os caminhoneiros não cuidam de sua saúde de forma adequada, associada a uma alimentação de má qualidade; uso de tabaco, álcool e rebite; e prática sexual

${ }^{1}$ Enfermeiro. Graduado em Enfermagem pela Universidade Federal de Mato Grosso. Barra do Garças, Mato Grosso, Brasil. E-mail: busanellorodrigo1@gmail.com ORCID ID: https://orcid.org/0000-00024754-9098

${ }^{2}$ Graduando em Farmácia. Universidade Federal de Mato Grosso (UFMT), Campus Universitário do Araguaia (CUA). Barra do Garças, Mato Grosso, Brasil. E-mail: edsonkysume19@gmail.com ORCID ID: https://orcid.org/0000-0002-9068-3700

${ }^{3}$ Graduando em Biomedicina. Universidade Federal de Mato Grosso. Barra do Garças, Mato Grosso, Brasil. E-mail: andre c v@hotmail.com ORCID ID: https://orcid.org/0000-0001-9254-642X

${ }^{4}$ Enfermeira. Graduado em Enfermagem pela Universidade Federal de Mato Grosso. Barra do Garças, Mato Grosso, Brasil. E-mail: marianasantana hbg@hotmail.com ORCID ID: https://orcid.org/0000-00033176-1171

${ }^{5}$ Enfermeiro. Doutor em Enfermagem. Universidade Federal de Santa Catarina (UFSC). Porto Alegre, Rio Grande do Sul, Brasil. E-mail: fernandoriegel85@gmail.com ORCID ID: https://orcid.org/0000-0002$\underline{3688-419 X}$

${ }^{6}$ Enfermeira. Doutora em Ciências pelo Programa de Pós-Graudação em Enfermagem Psiquiátrica da EERP/USP. Docente no curso de enfermagem da UFMT/CUA. Pontal do Araguaia, Mato Grosso, Brasil. Email: alisseia@hotmail.com ORCID ID: https://orcid.org/0000-0001-6155-6473

${ }^{7}$ Enfermeiro. Mestre em Ciências da Saúde. Docente no curso de enfermagem da UFMT/CUA. Barra do Garças, Mato Grosso, Brasil. E-mail: eliasufmt@yahoo.com.br ORCID ID: https:// https://orcid.org/00000002-0086-8286 Autor principal - Endereço para correspondência: Avenida Valdon Varjão, n. 6390, Barra do Garças, Mato Grosso, Brasil, CEP: 78600-000.

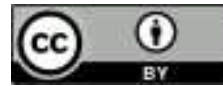

Este artigo está licenciado sob forma de uma licença Creative Commons Atribuição 4.0 Internacional, que permite uso irrestrito, distribuição e reprodução em qualquer meio, desde que a publicação original seja corretamente citada. 
insegura. Para a maioria dos caminhoneiros, o desempenho sexual foi considerado como satisfatório, embora de forma contraditória reportaram ejaculação precoce, dificuldade de manter a ereção e necessidade do uso de estimulantes sexuais. Conclusão: a sexualidade dos caminhoneiros pode estar comprometida devido a construção social da masculinidade. Por ser um tema permeado por preconceitos e tabus, os motoristas podem ter omitido aspectos em relação a sua sexualidade.

Descritores: Sexualidade; Saúde do Homem; Vulnerabilidade em Saúde.

\section{ABSTRACT}

Objective: to describe the behavior and sexuality aspects of truck drivers. Method: cross-sectional and quantitative study with 177 truck drivers in 2018, in a municipality in eastern Mato Grosso. Data collection was performed through semi-structured interviews. For data analysis and tabulation, descriptive statistics and Epi Info software were used. Results: it was found that truck drivers do not take care of their health properly, associated with poor quality food; use of tobacco, alcohol and rivet; and unsafe sexual practice. For most truck drivers, sexual performance was considered satisfactory, although in a contradictory way they reported premature ejaculation, difficulty maintaining their erection and the need to use sexual stimulants. Conclusion: truckers' sexuality may be compromised due to the social construction of masculinity. Because it is a theme permeated by prejudices and taboos, drivers may have omitted aspects in relation to their sexuality.

Descriptors: Sexuality; Men's Health; Health Vulnerability.

\section{RESUMEN}

Objetivo: describir los aspectos de comportamiento y sexualidad de los camioneros. Método: estudio transversal del enfoque cuantitativo con 177 camioneros que participaron en el proyecto "Live Well Trucker" en 2018, en un municipio del leste de Mato Grosso. La recogida de datos se realizó a través de entrevistas semiestructuradas. Para el análisis y tabulación de datos, se utilizó el software Epi Info. Resultados: se descubrió que los camioneros no cuidan su salud adecuadamente, asociados con alimentos de baja calidad; uso de tabaco, alcohol y remaches; y práctica sexual insegura. Para la mayoría de los conductores de camiones, el desempeño sexual se consideró satisfactorio, aunque de forma contradictoria informaron eyaculación precoz, dificultad para mantener su erección y la necesidad de usar estimulantes sexuales. Conclusión: la sexualidad de los camioneros puede verse comprometida debido a la construcción social de la masculinidad. Debido a que es un tema impregnado de prejuicios y tabúes, los conductores pueden haber omitido aspectos relacionados con su sexualidad.

Descriptores: Sexualidad; Salud del Hombre; Vulnerabilidad en Salud.

INTRODUÇÃO

Os caminhoneiros são

trabalhadores que deixam 0

conforto e o amparo do lar e 
frequentemente se distanciam da família, passando por uma diversidade de situações, dentre elas, estradas precárias e perigosas, prazos abusivos para entrega de cargas, violência no trânsito, estresse, solidão e remuneração pouco compatível com os riscos e desgastes que vivenciam.

Esses profissionais, em sua maioria, não possuem horários previstos para refeição e descanso, bem como pouco se preocupam ou conseguem cuidar da saúde. Normalmente, estão alheios à supervisão e assistência de serviços voltados à prevenção de doenças e promoção à saúde 1 , ficando mais vulneráveis ao adoecimento. Nesse cenário, discutir a sexualidade é de extrema relevância, especialmente por evidenciar a dificuldade em manter a regularidade com todos os hábitos saudáveis frente a saúde sexual.

Essa temática junto aos caminhoneiros tem uma abordagem recente. Anteriormente, a discussão acerca do tema era limitada somente às Infecções Sexualmente Transmissíveis (IST), sendo a classe abordada como grande disseminadora de tais agravos. Os estudos sobre os aspectos sexuais de caminhoneiros no Brasil tiveram início ainda no século $X X$, quando houve o rápido crescimento de casos de HIV no país ${ }^{1,2}$.

Pesquisa realizada na Índia com caminhoneiros mencionou que eles experimentam rotineiramente várias formas de privação material e simbólica, típico da vida às margens da sociedade, sendo relevante verificar até que ponto as intervenções em saúde junto ao grupo, com destaque na comunicação da mudança de comportamentos, poderão ter resultados efetivos ${ }^{3}$.

Uma revisão sistemática destacou que a ênfase na criação de hábitos saudáveis reflete a difusão de campanhas preventivas, que pressupõe que os bons resultados de saúde são uma junção de atitudes, comportamentos e indivíduos racionais e autônomos com capacidade de fazer escolhas responsáveis ${ }^{4}$. No entanto, os caminhoneiros possuem estilos de vida peculiar, vivendo e aderindo ao modelo hegemônico de masculinidade tóxica, que omiti a 
fragilidade e riscos de adoecer, desprivilegiando momentos de aprendizado, como por exemplo, em atividades de educação em saúde.

Assim, algumas necessidades em saúde, especialmente envolvendo a sexualidade não são atendidas e/ou compreendidas adequadamente e somam-se como fator para ampliar a vulnerabilidade deste grupo $^{5}$. Nesse panorama, questiona-se: quais as particularidades da sexualidade entre esses homens? A partir disso, o estudo teve como objetivo descrever

0 comportamento e os aspectos da sexualidade de caminhoneiros.

\section{MÉTODO}

Trata-se de estudo transversal e com abordagem quantitativa, junto a 177 homens caminhoneiros. 0 estudo foi realizado em postos de venda de combustíveis, no município de Barra do Garças (MT), Brasil, entre abril e setembro de 2018. Esse município está inserido em um cenário com grande fluxo de caminhoneiros devido a região estratégica para o escoamento e distribuição da produção agrícola e agropecuária do estado.

Foram estabelecidos critérios de inclusão e exclusão, sendo inclusos caminhoneiros do sexo masculino e excluídos aqueles com menos de seis meses de atuação ininterruptas.

A coleta de dados ocorreu durante $\mathrm{o}$ ano de execução do projeto de extensão "Viva bem Caminhoneiro" da Universidade Federal de Mato Grosso (UFMT), Campus do Araguaia, por aplicação de questionário contendo questões fechadas que abordaram aspectos sociodemográficos, profissionais e sexuais, aplicado pelos membros do projeto de extensão, devidamente treinados. Os caminhoneiros eram abordados e orientados a participar de uma roda de conversa sobre saúde sexual, porém anterior a conversa, eram convidados a participar do estudo, e os que aceitavam a participar, após assinar o TCLE eram entrevistados pelos pesquisadores e auxiliares de pesquisa. 0 instrumento de pesquisa foi aplicado em forma de entrevista, no qual o entrevistador preenchia o questionário de acordo 
com as respostas do participante. Logo após a entrevista, os participavam eram conduzidos para o espaço da roda de conversa sobre sexualidade.

Após a aplicação do questionário, os dados foram organizados em planilhas do Excel e analisados pelo programa Epi Info versão 3.5.1, através de estatística descritiva simples, utilizando números absolutos e relativos.

Durante este estudo não houve a separação dos conceitos de ejaculação e orgasmo no momento da entrevista, ficando atrelado apenas ao aspecto da ejaculação.

O estudo compõe projeto matricial intitulado: "Interface do cuidado: ressignificando as masculinidades frente ao ensino e a implementação de ações de promoção e prevenção à saúde do homem", aprovado pelo Comitê de Ética em Pesquisa com Seres Humanos (CEP/UFMT), sob número CAAE: 65604317.2.0000.5587, respeitando a Resolução n466/12 do Conselho Nacional de Saúde (CNS).

\section{RESULTADOS E DISCUSSÃO}

Os participantes do estudo possuíam entre 21 e 70 anos (média 37,5 anos), com baixa escolaridade, em união estável ou matrimônio e com filhos, renda mensal de um a cinco salários mínimos e religião cristã. Atuam como caminhoneiros de 11 a 20 anos, com jornada de trabalho entre 8 e 24 horas/dia.

Em relação a baixa escolaridade, estudo realizado na região do Vale do Araguaia $^{6}$, verificou que a maioria de caminhoneiros (59\%) tinha o ensino fundamental completo e somente $25 \%$ com ensino médio completo. Já em outro estudo realizado na região de Campinas (SP) ${ }^{1}$ o tempo de estudo foi inferior a oito anos para $64,8 \%$ destes profissionais.

Autores também têm mencionado que a baixa escolaridade de caminhoneiros e o menor acesso aos serviços de saúde, podem contribuir para diminuição do conhecimento acerca da função sexual, posto que possuem muitos mitos, tabus e conceitos errôneos ${ }^{7}$.

No Brasil, os motoristas dirigem em média 12,97 horas/ dia $^{8}$, corroborando com a presente pesquisa, em que $74 \%$ dirige entre 10 a 15 horas diárias. Além do 
excesso de horas trabalhadas

diariamente, observou-se no

presente estudo que esses

caminhoneiros passam muitos dias consecutivos na estrada, e que mais de $50 \%$ ficam de 15 a 30 dias fora de casa (Tabela 1).

Tabela 1 - Distribuição das horas diárias trabalhadas e dos dias fora de casa em decorrência do trabalho pelos caminhoneiros. Abril a setembro de 2018. Barra do Garças MT, Brasil. ( $n=177)$

Descrição

n

22

49

48

34

24

177

Total

Dias fora de casa

Até 5 dias

6 a 10 dias

11 a 15 dias

16 a 20 dias

21 a 25 dias

26 a 30 dias

31 ou mais dias

Total

Um estudo reportou que as relações extraconjugais estão relacionadas com a quantidade de dias longe de casa, sendo mais evidente conforme o tempo que esse motorista permanece nas estradas ${ }^{8}$.

Destaca-se ainda, que esse tempo em viagem e percorrendo longas distancias tende a ampliar o acesso e contato com diversidade de pessoas, inclusive com profissionais do sexo, que nestas circunstâncias são alternativas que muitos caminhoneiros buscam para suprir a solidão, problemas familiares e novas formas de comportamento sexual.

Neste estudo, a maioria dos caminhoneiros (65\%) assumiram a ocorrência de relações extraconjugais ou multiplicidade de parcerias sexuais. Outro estudo brasileiro, apontou essas relações 
em menor proporção $(16,5 \%)^{10}$.

Os caminhoneiros

demonstraram pouca preocupação

com sua saúde sexual, ao ponto que
$60 \%$ destes afirmaram não fazer uso de preservativo quando mantêm relações sexuais casuais em suas viagens.

Tabela 2 - Distribuição das características do comportamento de saúde e sexual dos caminhoneiros. Abril a setembro de 2018. Barra do Garças - MT, Brasil. $(n=177)$

\begin{tabular}{lcc}
\hline Descrição & $n$ & $\%$ \\
Relações sexuais extraconjugais & 115 & $65 \%$ \\
Sim & 62 & $35 \%$ \\
Não & 177 & 100 \\
Total & & \\
Uso do preservativo Masculino & 9 & $5 \%$ \\
Quase sempre & 62 & $35 \%$ \\
As vezes & 106 & $60 \%$ \\
Não faz uso & 177 & 100 \\
Total & & $40 \%$ \\
Motivo do uso do preservativo Masculino & 70 & $20 \%$ \\
Decisão própria & 36 & $4 \%$ \\
Decisão do casal & 7 & $26 \%$ \\
Decisão do parceiro(a) & 46 & $10 \%$ \\
Não faz uso & 18 & 100 \\
Não respondeu & 177 & \\
Total & &
\end{tabular}

\begin{tabular}{|c|c|}
\hline Quanto ao uso & com "mulheres limpas" e pela \\
\hline preservativo, os & confiança na proteção divina ${ }^{6}$. \\
\hline corroboram com estudo realizado & Diferentemente, outro \\
\hline em Teresina $(\mathrm{PI})$, enfatiza & apontou que $86 \%$ dos motor \\
\hline $45,8 \%$ dos caminhoneiros nunca & que referiram par \\
\hline faziam uso & faziam uso \\
\hline justificando essa aversão por não & preservativ \\
\hline $\operatorname{ar} \mathrm{da} s$ & realizada \\
\hline onibilida & \\
\hline
\end{tabular}


preservativos foi considerado entre seus entrevistados como fundamental, sendo o uso motivado principalmente pela preocupação da saúde da parceria fixa e não consigo próprio ${ }^{12}$.

Por outro lado, é importante destacar que o não uso do preservativo pode acarretar diversos problemas, como a possibilidade de gravidez indesejada/ paternidade irresponsabilidade ou distante, conflitos nas relações com a parceria definida como principal, repercussões quanto aos aspectos sociais e psicoemocionais, além da exposição às infecções e doenças.

Outro estudo realizado em Itabaiana (SE) e com caminhoneiros no sudoeste da Bahia (BR), também apontou que a relação sexual desprotegida é o principal fator que amplia o risco para adquirir $\mathrm{HIV} /$ AIDS $^{11}$.

Um estudo realizado na Tanzânia destacou que as condições de trabalho (longos períodos parados esperando liberação das cargas e longos períodos longe da parceria fixa), consumo de drogas (álcool), influência dos colegas e a venda facilitada de sexo, acabam por influenciar a atividade de risco sexual dos caminhoneiros ${ }^{14}$.

Em contrapartida, estar com alguma IST, irá requerer destes caminhoneiros tempo para buscarem por tratamento ${ }^{15}$, e quando isso não ocorrer, devido a sua dinâmica de trabalho, ou ocorrer de forma inadequada, como procurar por medicações em farmácias ou por indicação de colegas, poderá comprometer ainda mais a sua saúde ${ }^{8}$.

Em se tratando da gravidade que 0 não uso de preservativo pode acarretar na vida dos caminhoneiros, torna-se necessário que profissionais da saúde elaborem estratégias preventivas. Um estudo destacou que essas estratégias podem ser do cotidiano do caminhoneiro como, por exemplo, aconselhar este homem a se auto masturbar, a expor fotos e roupas íntimas femininas no painel do caminhão, a fim transmitir a mensagem da presença de parceria a bordo e existência de família ${ }^{14}$.

Tais orientações parecem comuns, porém são acessíveis e práticas à vida de um caminhoneiro, e assumem caráter 
de atividades de promoção do autocuidado.

Aspectos da sexualidade dos caminhoneiros

Os caminhoneiros

reproduzem um comportamento sexual influenciado pelas gerações anteriores. A sexualidade e práticas sexuais normalmente estão atreladas a desmedida influência de pares, mídia e entretenimento com conteúdo adulto ${ }^{4}$.

Além disso, o estereótipo que vulgariza a sexualidade apenas como $o$ ato sexual, protagonizando que o homem é um sujeito viril e que a sua satisfação sexual depende unicamente da performance erótica, é altamente difundido na sociedade, comprometendo o viver saudável da sexualidade.

No presente estudo, 0 desempenho sexual foi auto avaliado como ótimo ou bom por 79\% dos entrevistados (Gráfico 1). Verifica-se ainda no gráfico 2, que os participantes estão realizados com seu desempenho sexual, seja no interesse sexual (65\%), autoconfiança para seduzir (41\%) e presença frequente de orgasmos sexuais $(81 \%)$.

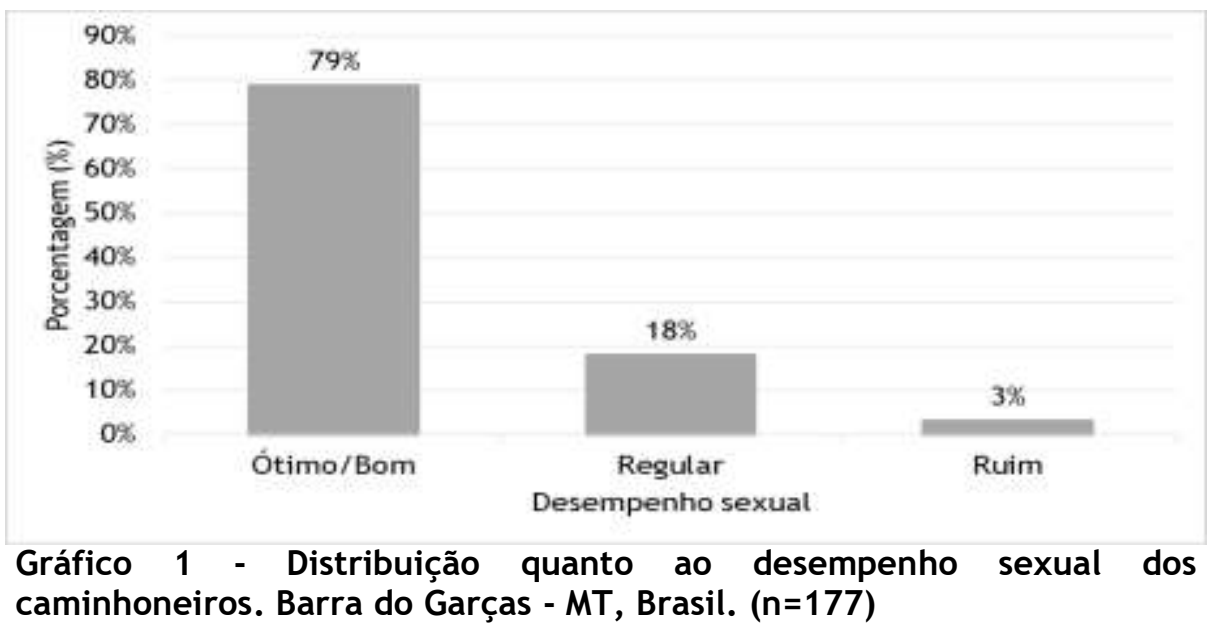




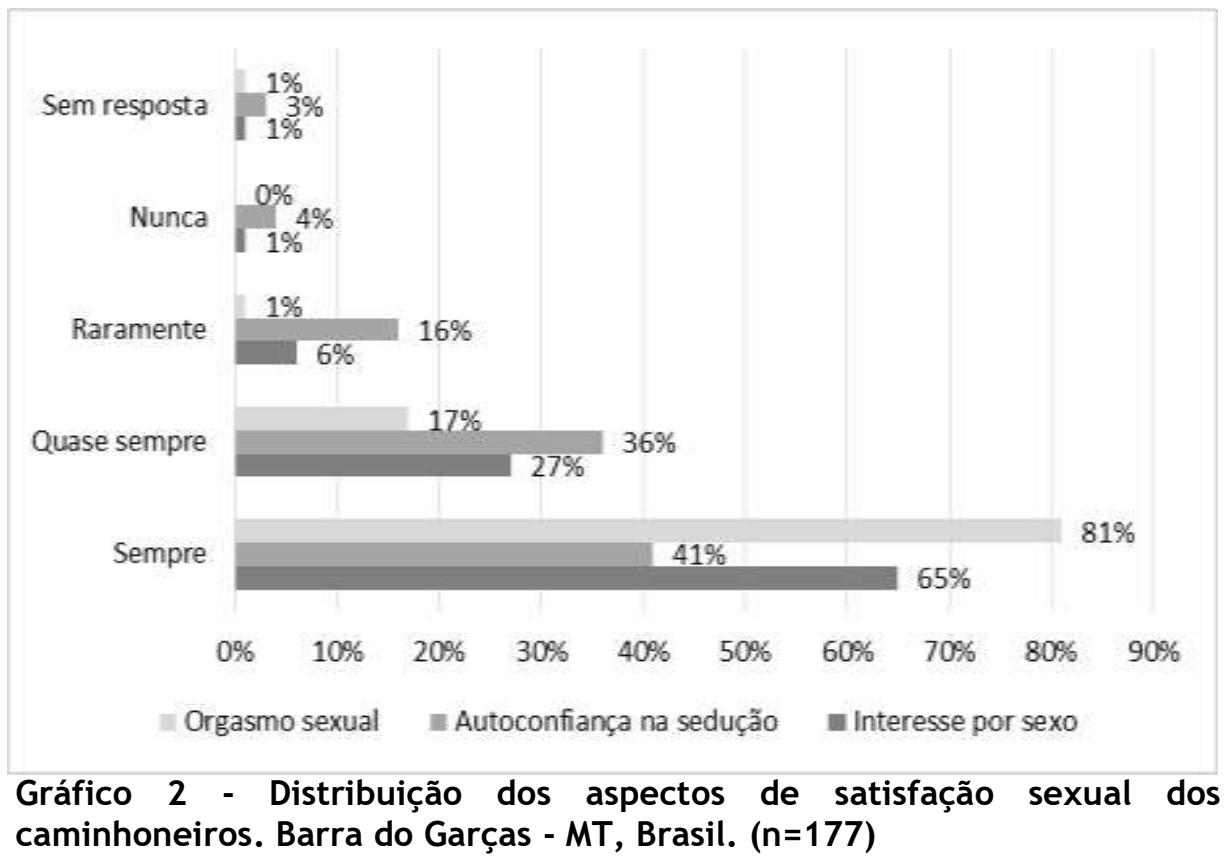

Um estudo realizado em São Outra característica sexual Paulo (BR) revelou que a maioria dos homens $(91,3 \%)$ estavam satisfeitos com seus relacionamentos, classificando-os como bons ou excelentes, sentindo atração sexual por suas parcerias $(97,3 \%)$, o desejo sexual era excelente ou bom (93\%) e que todos se sentiam sexualmente potentes ${ }^{16}$.

Embora os caminhoneiros da presente pesquisa tenham demonstrado satisfeitos com a performance sexual, a cabine ou boleia do caminhão, local onde $o$ ato sexual muitas vezes é realizado, não apresenta um ambiente seguro e adequado, 0 que pode afetar diretamente no desempenho sexual desses homens, pela falta de conforto e privacidade ${ }^{17}$. abordada nesse estudo foi a ereção, controle da ejaculação e o uso de estimulantes sexuais. A ejaculação precoce esteve presente entre mais de $50 \%$ dos caminhoneiros (Gráfico 3).

Semelhantemente a esta pesquisa, a disfunção erétil também esteve presente entre os participantes de estudo em São Paulo ${ }^{16}$ e no Rio Grande do Sul ${ }^{17}$.

A disfunção erétil pode estar ligada a diversos fatores como a obesidade, dislipidemia, uso de drogas, a idade, falta de interesse, a questão hormonal. 0 sedentarismo relatado pelos caminhoneiros é um influenciador na disfunção. Um estudo relacionou a prática de exercício físico e a 
Busanello RS, Lima EAS, Vilela AC, Santana MR, Riegel F, Lemes AG, et al. Perfil de comportamento... disfunção erétil, demonstrando que elevada intensidade, há uma no grupo ativo que pratica melhor função erétil em relação exercício aeróbico de moderada a aos sedentários ${ }^{17}$.

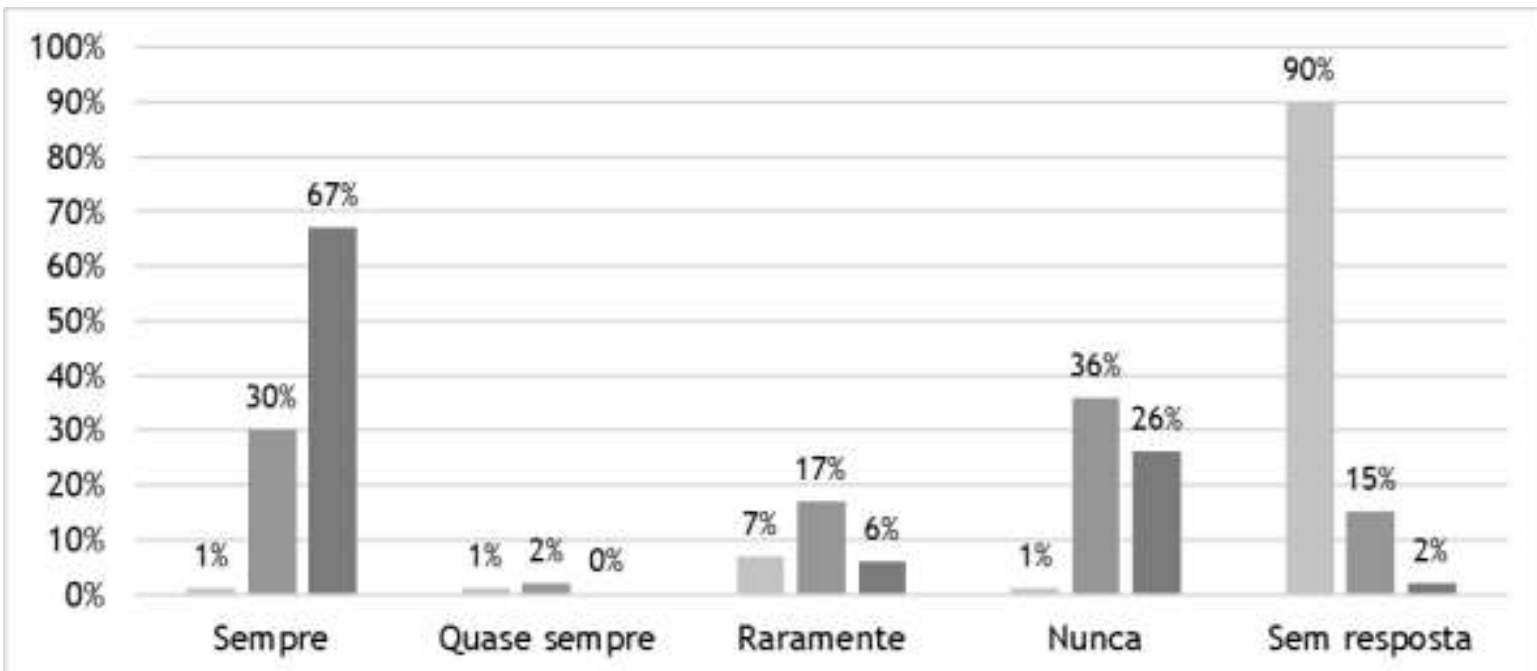

Estimulantes sexuais = Controle da ejaculação | Mantêm o pênis ereto durante o sexo

Gráfico 3 - Aspectos da sexualidade como a ereção durante o sexo, controle da ejaculação e uso de estimulantes sexuais. Barra do Garças (MT), Brasil. ( $n=177)$

Estudo brasileiro apontou

que poucos homens realizam tratamento específico para disfunção erétil, com uso de medicamentos orais ${ }^{17}$.

0 uso de estimulantes muitas vezes serve como segurança psicológica para os homens. A droga aumenta a autoconfiança e otimiza consecutivamente a performance sexual ${ }^{15}$.

Ambos os fatores

mencionados devem sinalizar aos profissionais a importância de conhecer e compreender as condições de vida e os desdobramentos na sexualidade dos caminhoneiros, ao entende-los como um grupo de maior vulnerabilidade e que requer ações contínuas de prevenção e intervenção.

Para além do compromisso dos profissionais de saúde, a educação sexual promovida nos diversos contextos da comunidade, escolas, igrejas, praças e campos de futebol fortalecem o processo educativo, dissemina uma nova cultura de cuidado e tende a desenvolver melhor nível de literacia em saúde ${ }^{18}$.

Outra estratégia, volta-se a criação de espaços que visem 
dialogar sobre os mitos que envolvem a sexualidade e que interferem na vida sexual desses caminhoneiros $^{16}$.

\section{CONSIDERAÇÕES FINAIS}

No estudo, o ambiente não era propício para o debate de fatores libidinosos e da sexualidade, por ser aberto e sem privacidade, porém corresponde ao espaço em que concentrava essa clientela. Esse aspecto pode ter interferido nas respostas, sendo a principal limitação do estudo.

A dinâmica do trabalho dos caminhoneiros, em decorrência da carga horária diária, quantidade de dias distantes de casa e família, bem como aspectos particulares e da cultura masculina, aproxima-os do contato com profissionais do sexo, adesão a práticas sexuais de risco (sexo sem proteção) e uso de estimulantes sexuais. Apesar disso, sentem-se atraídos por suas parceiras e estão realizados sexualmente.

A sexualidade destes caminhoneiros parece estar comprometida, tanto pela falta de assistência/atenção profissional como pela construção social da masculinidade e da rotina de trabalho que possuem.

Frente a estes achados, é fundamental mobilizar as equipes de saúde dessa localidade, para estabelecerem projetos terapêuticos aplicáveis a esse estilo de vida, pouco frequente nos serviços de saúde, mas que carece de múltiplas necessidades, especialmente sexuais.

\section{REFERÊNCIAS}

1. Masson VA, Monteiro MI. Estilo de vida, aspectos de saúde e trabalho de motoristas de caminhão. Rev Bras Enferm. 2010; 63(4):533-540.

2. Silva AL, Maia ACCO. A evolução da sexualidade masculina através do tratamento da ejaculação precoce sob a luz da terapia cognitivo-comportamental. Cad UniFOA. 2017; 3(1):87-96.

3. Sastry S. Long distance truck drivers and the structural context of health: a culturecentered investigation of indian truckers' health narratives. Health Commun. 2016; $31(2): 230-241$. 
4. Sastry S, Dutta MJ. Global health interventions and the "common sense" of neoliberalism: a dialectical analysis of pepfar. J Int Intercult Commun. 2013; 6:21-39.

5. Juneja S, Rao Tirumalasetti V, Mishra RM, Sethu S, Singh IR. Impact of an HIV prevention intervention on condom use among long distance truckers in India. AIDS Behav. 2013; 17:1040.

6. Araújo TME, Rocha DRF, Silva AAS, Souza KAA, Chaib NL. Comportamentos sexuais e fatores associados entre caminhoneiros de rota longa. Rev Enferm UFPI. 2015; 4(2):25-32.

7. Rocha EM, Lemes AG, Santos BLM. Exposição de caminhoneiros à infecções sexualmente transmissiveis. J Health NPEPS. 2017; 2(1):230-240.

8. Rocha EM, Siqueira MFC, Santos BLM, Silva MHP. Prevalência de obesidade e sedentarismo em caminhoneiros. Rev Elet Inter. 2015; 13(1):165-169.

9. Alessi A, Alves MK. Hábitos de vida e condições de saúde dos caminhoneiros do Brasil: uma revisão da literatura. Ciênc Saúde. 2015; 8(3):129-136.

10. Costa PF, Cerqueira-Santos E. Fatores associados ao uso de preservativo e relações com prostitutas entre caminhoneiros do Brasil. Psicol saúde doenças. 2018; 19 (3):617-627.

11. Rohden F. Diferenças de gênero e medicalização da sexualidade na criação do diagnóstico das disfunções sexuais. Rev estud fem. 2009; 17(1):1-21.

12. Sobrinho-Santos CK, Silva AV, Malheiros AF, Trindade RA, Pagan AA. Relatos de caminhoneiros sobre a prevenção do HIV e o material educacional impresso: reflexões para educação em saúde. Ciênc educ. 2015; 21(4):1011-1030.

13. Sousa LMS, Silva LS, Palmeira AT. Representações sociais de caminhoneiros de rota curta sobre HIV/AIDS. Psicol Soc. 2014; 26(2):346-355.

14. Kohli A, Kohli A, Kerrigan D, Brahmbhatt $\mathrm{H}$, Likindikoki S, Beckham J, Mwampashi A, et al. Social and structural factors related to HIV risk among truck drivers passing through the 
Iringa region of Tanzania. AIDS Care. 2017; 29(8):957-960.

15. Nascimento NS, Leitão LNO, Santos MWS, Silva AKC, Pereira LM, Teles FA. Ejaculação precoce: existe tratamento? Rev saúde desen. 2014; 5(3):180-195.

16. Reis MMF, Glina $S$, Abdo CHN. Percepções sobre o tamanho do pênis em homens brasileiros supostamente saudáveis de $40 \mathrm{a}$ 60 anos: um estudo piloto transversal. Sao Paulo Med J. 2015; 133(2):84-90.
17. Duarte DV, Restrepo-Méndez MC, Silveira MF. Prevalence of erectile dysfunction oral drugs use in a city of southern Brazil. Ciênc Saúde Colet. 2017; 22(8):2763-2770.

18. Sawal N, Hans GDR, Verma G. Sexual practices, myths and misconceptions among long distance truck drivers in North India. QJM: Int J Med. 2016; 109:467-472.

Conflito de interesses: Os autores declaram não haver conflito de interesses.

\section{Participação dos autores:}

- Concepção: Busanello RS, Lima EAS, Vilela AC, Santana MR, Riegel F, Lemes AG, Rocha EM.

- Desenvolvimento: Busanello RS, Lima EAS, Vilela AC, Santana MR, Riegel F, Rocha EM.

- Redação e revisão: Busanello RS, Lima EAS, Vilela AC, Santana MR, Riegel F, Lemes AG, Rocha EM.

Como citar este artigo: Busanello RS, Lima EAS, Vilela AC, Santana MR, Riegel F, Lemes AG, et al. Perfil de comportamento e características sexuais de caminhoneiros. J Health NPEPS. 2020; 5(1):228-241. 